\title{
Cultura organizacional en empresa de venta de productos de lunchería
}

Organizational culture in company of sale of lunchery products

Casimiro, Willsany

willsany@hotmail.com

Código ORCID: 0000-0001-8810-0951

Universidad de Carabobo, Venezuela
Edumary Medina

edumarymedina@gmail.com

Código ORCID: 0000-0001-5098-3031

Universidad de Carabobo, Venezuela

Artículo recibido septiembre 2019 / Arbitrado en octubre 2019 / Publicado en enero 2020

RESUMEN

ABSTRACT
El presente estudio tiene como objetivo evaluar la cultura organizacional de la empresa C.M., C.A. ubicada en Valencia, estado Carabobo, Venezuela que permita sentar las bases para recomendar estrategias dirigidas a mejorar el clima y desempeño de la organización. Para lograr esto se definió como objetivos específicos diagnosticar la cultura organizacional de la empresa y examinar la cultura organizacional existente. La metodología empleada es un enfoque cuantitativo con diseño de campo, no experimental. Se aplicó un cuestionario a diez trabajadores de C.M., C.A. de quince ítems con respuestas de tipo policotómicas. Los resultados analizados en base a las bases teóricas consultadas, permitieron evidenciar de manera concluyente que la empresa presenta debilidades en su cultura organizacional.

Palabras clave: Estrategias, valores, cultura organizacional

The objective of this study is to evaluate the organizational culture of the company C.M., C.A. located in Valencia, Carabobo state, Venezuela that allows laying the foundations to recommend strategies aimed at improving the climate and performance of the organization. To achieve this, specific objectives were defined to diagnose the organizational culture of the company and examine the existing organizational culture. The methodology used is a quantitative approach with field design, not experimental. A questionnaire was applied to ten C.M., C.A. of fifteen items with polycotomic responses. The results analyzed based on the theoretical bases consulted allowed conclusive evidence that the company has weaknesses in its organizational culture.

Kev words: Strateaies, values, oraanizational culture 


\section{INTRODUCCIÓN}

Actualmente, la globalización ha abarcado un proceso de creciente internacionalización del capital humano, debido al uso de nuevas políticas internacionales, surgimiento de nuevos procesos y uso intensivo de la tecnología sin precedentes. Es por ello, que los individuos y las organizaciones empresariales desean conservar y preservar sus creencias, culturas y valores, a fin de evitar perder su propia identidad y a su vez mejorar su competitividad y desempeño.

Es así como, mundialmente se ha evidenciado la puesta en práctica de varias tendencias económicas y sociales, las cuales están causando un gran impacto en la cultura organizacional y en las maneras de coordinar y motivar a los trabajadores, para aumentar el valor que las organizaciones puedan generar. En este sentido, las organizaciones en el mundo enfrentan grandes desafíos, los cuales repercuten en lo administrativo, competitividad, efectividad de manejar la diversidad cultural y adaptación a los cambios políticos, sociales y económicos que se generen en el entorno.

En lo que concierne a América Latina, los sistemas organizacionales que existen, y los que se creen dentro de esta región, sólo pueden sobrevivir si son coherentes con las organizaciones. Esto significa, que necesariamente tienen como parte importante de sus premisas de decisión, factores propios de la cultura de Latinoamérica. Tanto si es una organización transnacional, que instala una nueva filial en algún país latinoamericano, como si se trata de una organización gubernamental creada para enfrentar algún problema derivado de las necesidades del desarrollo o si se está hablando de una pequeña organización, de tipo comercial $\mathrm{o}$ industrial, todas ellas tienen incorporadas en su accionar, premisas de decisión provenientes de la cultura local.
Así pues, son varias las razones por las cuales se debe fomentar la cultura organizacional, entre ella adaptarla a los cambios de la época para lograr el éxito. Además, el desarrollo de la cultura organizacional permite a los integrantes de la organización ciertas conductas e inhiben otras. Una cultura laboral abierta y humana alienta la participación y conducta madura de todos los miembros de la organización, sí las personas se comprometen y son responsables, se debe a que la cultura laboral se lo permite, es una fortaleza que encamina a las organizaciones hacia la excelencia, hacia el éxito. Al respecto, Robbins (2004) señala que "en una cultura fuerte, los valores centrales de la organización son sostenidos con firmeza, y son muy compartidos". (p. 527).

En este orden de ideas, Chiavenato (2004) afirma que "la cultura atrae la atención, transmite una visión y por lo general honra como héroes a los individuos productivos y creativos" (p. 108). Dicha cultura describe el ambiente interno incorporando aquellos: supuestos, creencias y valores; que compartidos por todos los integrantes guían y dirigen su funcionamiento y si son fuertes y estratégicamente correctos repercuten en el éxito a largo plazo de la organización. Desde este punto de vista, la cultura organizacional para que propicie cambios en el comportamiento de los empleados, debe alinearse a un sistema social complejo de leyes, valores, costumbres y creencias, a fin de generar una interacción entre los empleados y su ambiente.

Se debe resaltar que, para alcanzar solidez en la cultura organizacional, se deberán aplicar estrategias, las cuales surgen de tomar los elementos que la sociedad, durante siglos y años, ha ido enriqueciendo y pasando de generación en generación. La estrategia es el resultado del proceso de planeación; pero, a su vez, es la base 
para una correcta organización, dirección, control y evaluación. Es por ello, que la humanidad ha seguido hablando de estrategias y la historia recoge que los grandes hombres siempre se han planteado grandes estrategias.

Este es el caso la empresa C.M., C.A. dedicada a la venta de lonchería ubicada en Valencia estado Carabobo, constituida como una pequeña empresa, cuyo objeto es la comercialización al mayor y detal de todo tipo de desayunos específicamente empanadas, arepas rellenas, jugos, lácteos, chucherías, entre otras cosas. En dicha empresa se ha observado una serie de debilidades, entre éstas una gerencia centralizada que, por su condición de pequeña empresa, no se delegan funciones, y éstas recaen en una persona; de igual manera la visión, misión y metas no están establecidas, los trabajadores no saben hacia dónde deben dirigirse y cuáles son las prioridades dentro de la empresa, para la consecución de sus objetivos; igualmente no están definidos los valores que la identifique como organización.

Dentro de este marco de ideas, el presente estudio tiene objetivo general, evaluar la cultura organizacional de la empresa C.M., C.A. ubicada en Valencia, estado Carabobo, Venezuela, con la finalidad de recomendar estrategias dirigidas a mejorar el clima y desempeño de la organización. Para lograr esto se definieron como objetivos específicos diagnosticar la cultura organizacional de la empresa y examinar la cultura existente de la misma.

Para que toda empresa responda satisfactoriamente a sus objetivos organizacionales, tiene que estar sujeta a una constante evaluación para lograr distinguir que la dinámica general del cambio en la sociedad actual crea desajustes, lo cual hace que se reconozca cada vez y con mayor interés en las empresas modernas, la influencia que tiene el clima organizacional con el bienestar o el buen rumbo de la gerencia en su funcionamiento administrativo, el cual se encuentra englobado en la expresión Cultura Organizacional, como variable relevante en el rendimiento laboral.

Por lo tanto, para que una organización pueda cambiar funcionalmente, debe evaluar su cultura organizacional y estar acorde a los objetivos de la organización, la cual es reconocida como un conjunto de valores, creencias y conductas; siendo lógico pensar que cuando cambia la estrategia organizacional cambia la cultura; entonces, lo que antes era un apoyo, ahora puede ser un obstáculo; de ahí que la gerencia efectiva de la cultura corporativa sea considerada, un elemento esencial en la implantación de una decisión estratégica.

Los resultados que se esperan de este estudio, podrán contribuir para que la empresa del caso propicie una cultura organizacional sólida dirigida a su recurso humano, para lograr alcanzar la mejor comunicación y el mayor sentido de pertenencia, lo cual le permita un desempeño de funciones de la manera más eficiente y eficaz posible, con el fin de llegar a alcanzar las metas y los objetivos propuestos por la organización. En relación a ello, se han de generar beneficios importantes que se manifestarán en una mejor gestión, tanto en la organización como en los trabajadores, debido a que por ser una empresa en crecimiento podrá atraer un mayor número de clientes, lo cual le producirá mayor rentabilidad y crecimiento organizacional interno.

Desde la relevancia social, la investigación permitirá que, por un lado, los empleados puedan lograr una máxima satisfacción laboral que impulsa la efectividad en su desempeño que conlleva a una mejor situación familiar; y por el otro, los clientes se verán beneficiados, ya que obtendrán un mejor servicio en lo que respecta a 
la atención por parte de la empresa y así poder satisfacer sus necesidades de la mejor manera posible.

\section{MATERIALES Y METODO}

En este apartado se aborda el marco metodológico, de vital importancia en toda investigación, pues mediante este procedimiento ordenado se permite establecer la secuencia de hechos y fenómenos dirigidos hacia el significado del estudio.

Este estudio se define como una investigación descriptiva de campo, con enfoque cuantitativo, basado en la recolección de datos directamente de los sujetos investigados. Se recolectó la información directamente del lugar donde suceden los hechos, de primera fuente, es decir en el área laboral de los trabajadores en estudio, las instalaciones de la empresa C.M., C.A. ubicada en Valencia estado Carabobo.

De allí que se tomaron a todos los empleados en estudio por ser una población pequeña. Por lo que la muestra censal está formada por todos los trabajadores de la empresa. Se utilizó como técnica de recolección de datos, la encuesta siguiendo una modalidad cuantitativa,

El instrumento usado dentro del enfoque cuantitativo, técnica encuesta, fue el cuestionario. En este particular, el cuestionario aplicado en esta investigación presenta las siguientes características: contiene quince (15) afirmaciones con respuestas de tipo policotómicas (Siempre, Casi Siempre, A Veces, Casi Nunca y Nunca) con la intención de diagnosticar la cultura organizacional de la empresa C.M., C.A.

La técnica de procesamiento y análisis de los resultados fue a través de un procesamiento estadístico descriptivo, detallado en tablas o cuadros de frecuencia y porcentaje, agrupados finalmente en gráficos de tipo columnas, con el propósito de organizarlos e intentar dar respuesta a los objetivos planteados, evidenciar los principales hallazgos, conectándolos de manera directa con los indicadores planteados en el cuadro técnico metodológico que sustentan la misma, así como, con los conocimientos que se disponen en relación al problema que se propone estudiar.

\section{RESULTADOS Y DISCUSION}

En el tratamiento de la información se sustentó la investigación, en la modalidad cuantitativa, por lo que los datos recogidos de la muestra en estudio, fueron organizados por indicadores, para un procesamiento estadístico descriptivo, manifiesto en tablas de frecuencia y porcentaje, agrupados en gráficos de columnas.

Los resultados obtenidos a través de cada alternativa de respuesta del cuestionario, se analizaron e interpretaron primero independientemente de manera específica y de manera global a través de cada indicador, con la intención de contrastar por medio de indicadores la opinión de cada uno de los integrantes de la población en estudio

Resultados del ítem 1: Elementos de la cultura Organizacional. Indicador: Medio ambiente: se pudo evidenciar que el cincuenta por ciento (50\%) de los trabajadores en estudio considera que casi siempre su comportamiento laboral está influenciado por el entorno, el cuarenta por ciento (40\%) señala que siempre influye y el restante diez por ciento (10\%) considera que solo es a veces influenciado. Estos resultados demuestran que el entorno de la empresa influye en el desarrollo laboral de los trabajadores. Coincidiendo estos resultados con lo que indica Chiavenato (1999), pues ciertamente el comportamiento está influenciado por el ambiente y por las diversas actividades y normas informales existentes en los diversos 
grupos. Esta teoría hace hincapié que solamente la comprensión de la naturaleza de estas relaciones humanas permite al jefe obtener mejores resultados de sus empleados, ya que logrando ésta, facilita una atmósfera en donde cada individuo es estimulado y motivado a expresarse libremente.

Cada organización debe enfrentarse a un entorno determinado, el cual puede variar, algo que puede incidir de manera considerable a que se de esta variación, es la dedicación o razón de ser de la empresa. La organización siempre debe tomar en cuenta el entorno, este puede estar dado por factores políticos, tecnológicos, económicos, de competencia, entre otros, todo esto de alguna manera puede causar gran influencia en la formación y estabilidad de la cultura organizacional.

Los factores externos y el ambiente donde se desenvuelve la empresa, va a ser un elemento determinante para que la organización obtenga el éxito deseado. Es por esto, que las organizaciones deben saber manejarse en distintos ambientes, y adaptarse a los cambios, ser competitiva, y la cultura que se establezca debe desarrollarse tomando en consideración estos aspectos importantes.

Resultados al ítem 2. Indicador: Factores externos: el sesenta por ciento (60\%) de los trabajadores considera que los factores externos siempre tienen incidencia en el rendimiento de la empresa, el treinta por ciento (30\%) indica que esto siempre es así y el restante diez por ciento (10\%) coincide en considerar que es solo a veces. Esto evidencia que la problemática de las relaciones humanas entre directivos, empleados y entorno, se debe argumentar en el enfoque humanístico de la teoría de las relaciones humanas en la organización, por lo que Chiavenato (1999) señala que los factores externos y el ambiente donde se desenvuelve la empresa, va a ser un elemento determinante para que la organización obtenga el éxito deseado. Es por esto que las organizaciones deben saber manejarse en distintos ambientes y adaptarse a los cambios, ser competitiva, y la cultura que se establezca debe desarrollarse tomando en consideración estos aspectos. Por lo que resulta importante tomar en cuenta el entorno en el cual se desarrollan las operaciones de la empresa, pues éste influye en las mismas, entonces para buscar mejoras se debe evaluar el entorno en búsqueda de una influencia positiva en la empresa C.M., C.A.

En relación al ítem 3, Indicador Valores, el cincuenta por ciento (50\%) de los trabajadores indicó que la empresa nunca posee valores definidos, el cuarenta por ciento (40\%) señalo que casi nunca y el diez por ciento (10\%) restante dice a veces. Situación desfavorable para la empresa, por lo que es necesario definir valores, los cuales son intangibles, pero entre los trabajadores son conocidos, estos van a establecer un sentido de dirección entre ellos, para obtener conductas y modos de comportamientos esperados. Gutiérrez (2004) señala que los valores se pueden definir como una serie de creencias, que se consideran buenas dentro de la organización, esta creencia es permanente y la misma va a contribuir para que se establezcan una serie de comportamientos que son calificados como aceptados dentro de la misma.

Los valores de acuerdo a Denison (1991) representan "la base de evaluación que los miembros de una organización emplean para juzgar situaciones, actos, objetos y personas." (p. 20). Estos reflejan las metas reales, así como, las creencias y conceptos básicos de una organización y, como tales, forman la médula de la cultura organizacional. Los valores según Deal y Kennedy (1985) "son los cimientos de cualquier 
cultura organizacional, definen el éxito en términos concretos para los empleados y establecen normas para la organización". (p. 19). Como esencia de la filosofía que la empresa tenga para alcanzar el éxito, los valores proporcionan un sentido de dirección común para todos los empleados y establecen directrices para su compromiso diario. Indica Robbins (2004) "Los valores inspiran la razón de ser de cada institución, las normas vienen a ser los manuales de instrucciones para el comportamiento de la empresa y de las personas". (p. 111).

Por lo tanto, toda organización con aspiraciones de excelencia debería tener comprendidos y sistematizados los valores y las ideas que constituyen el comportamiento motor de la empresa. En consecuencia, los planteamientos descritos anteriormente, conducen a pensar que los valores están explícitos en la voluntad de los fundadores de las empresas, en las actas de constitución y en la formalización de la misión y visión de las organizaciones. Los valores se generan de la cultura de la organización, por lo tanto, son acordados por la alta gerencia. Igualmente son exigibles y se toman en cuenta para la evaluación y el desarrollo del personal que integra la organización.

En relación a los resultados obtenidos en el ítem 4, Indicador Héroes organizacionales, el cincuenta por ciento (50\%) de los trabajadores indicó que a veces se reconoce en la empresa a algún empleado líder y promotor de buenas acciones, el cuarenta por ciento (40\%) señala que casi nunca se reconoce y el restante diez por ciento (10\%) señala que nunca. Siendo importante reconocer a este tipo de personal pues los héroes, son unas de las personas que se encargan de cumplir y hacer cumplir los valores organizacionales, ya que estos con su carácter y estilo, sobresalen. Al respecto, Deal y Kennedy (1985), plantean que los héroes pueden lograr que el éxito sea alcanzable, pues al trabajar toman en cuenta los principios organizacionales.

El análisis del ítem 5, Indicador: Visión. El cien por ciento (100\%) de los trabajadores en estudio indicó que no conoce la visión de la empresa. Situación desfavorable para la empresa, pues se evidencia la inexistente definición de la visión de la empresa. En relación a esto, se puede decir según Daft y Marcic (2006) que la visión se refiere a la percepción clara de un futuro deseado, un ideal para la organización. Se expresa en la mega estrategia; sin embargo, debe ser creíble y realista. Se puede afirmar que la visión institucional es una imagen compartida de lo que los individuos quieren hacer de la organización. La construcción de la visión es tarea de la alta gerencia, por lo que resulta importante ser definida en la propuesta de esta investigación

Resultados del ítem 6, Indicador: Misión. Se pudo evidenciar que el cien por ciento (100\%) de los trabajadores en estudio indicó que no se conoce la misión de la empresa. Siendo necesario definir la misión de la empresa pues, la misión de una organización es una declaración relativamente duradera del propósito de la misma; que la diferencia de otras y le da su razón de ser. Se traduce entre otras cosas en lo que sus empleados hacen todos los días, simbolizando su legitimidad para todos los factores que la rodean (Agentes internos y externos). Daft y Marcic (2006), enuncian Una misión sólida también tiene un impacto sobre los empleados, puesto que los habilita para que se comprometan con la organización.

Tanto la visión como la visión como la visión son factores claves para que los integrantes de una empresa tengan un desempeño proactivo, ya que la primera se refiere a la percepción clara 
de un futuro deseado, un ideal o un sueño para la organización. Se expresa en la mega estrategia; sin embargo, debe ser creíble y realista. Se puede afirmar que la visión institucional es una imagen compartida de lo que los individuos quieren hacer de las organizaciones; además, responde a la pregunta de ¿Cómo se desea que aquellos por los que existe la organización vean dicha institución? La construcción de la visión es tarea de la alta gerencia; así como, una pintura no es creada por un gran comité, una visión no puede ser creada por una multitud.

En referencia a la misión de una organización, es una declaración relativamente duradera del propósito de una organización; que la diferencia de otras y le da su razón de ser. Se traduce entre otras cosas en lo que sus empleados hacen todos los días, simbolizando su legitimidad para todos los factores que la rodean (Agentes internos y externos). Daft y Marcic (2006), enuncian: "Una misión sólida también tiene un impacto sobre los empleados, puesto que los habilita para que se comprometan con la organización" (p. 148). Siendo así, la misión institucional permite que los individuos se identifiquen con el propósito general y los ayuda a motivarlos, logrando un compromiso con el propósito organizacional. La misión debe identificar las necesidades que existen en el entorno social y prever tanto como, su posible solución y su evolución.

Una buena declaración de misión debe describir el propósito de la organización, la necesidad de sus clientes o usuarios, productos o servicios, mercado y filosofía. Dentro de las características de una buena declaración de misión se puede citar: Definir como es la organización y como querría ser. Debe ser específica en cuanto a expresar los límites del servicio y bastante amplia para dar cabida al crecimiento creativo. Diferenciar a la organización dentro de las demás. Servir de marco para evaluar las actividades presentes y futuras. Estar redactada en términos bastante claros como para que sea entendida fácilmente.

Respecto a los resultados del ítem 7, Indicador: Identidad organizacional. Los encuestados consideran en un cincuenta por ciento (50\%) que los trabajadores casi nunca tienen sentido de pertenencia para la empresa, un cuarenta por ciento (40\%) considera que a veces y el restante diez por ciento (10\%) dice que nunca. Resultados que hacen evidente la necesidad de aumentar el sentido de pertenencia en la empresa. Gutiérrez (2004) señala que la identidad organizacional debe estar impresa en todas partes donde se involucre la organización, una organización en la que sus individuos tienen poca identificación institucional difícilmente puede inspirar apego y sentido de pertenencia, para consecuentemente lograr los objetivos organizacionales. La identidad Organizacional: Se refiere a la percepción que la organización tiene sobre sí misma. Ésta es la suma de la historia de la organización con las estrategias y políticas de dirección. De este modo, la identidad organizacional debe estar impresa en todas partes donde se involucre la organización para así, tener una posición en el mercado y ser reconocida externa e internamente. Una organización en la que sus individuos tienen poca identificación institucional difícilmente puede inspirar apego y sentido de pertenencia.

Análisis: Indicador: Comunicación e información en la organización. En este particular, el sesenta por ciento (60\%) de los trabajadores indico que a veces existe comunicación fluida en la relación entre los trabajadores mientras el cuarenta por ciento (40\%) restante señalo que siempre hay comunicación. Por lo que se deben aplicar estrategias para hacer más fluida la comunicación 
en la empresa C.M., C.A. así, la comunicación es una herramienta fundamental en la práctica de la gerencia de una organización, se concibe incluso como fundamental para el éxito empresarial. Hampton citado por Rodríguez (2006), la define como el proceso mediante el cual las personas que trabajan en las organizaciones transmiten información entre ellas e interpretan correctamente su significado.

Análisis de la medición del Indicador: Motivación En este particular, cincuenta por ciento $(50 \%)$ de los trabajadores indicó que la empresa a veces motiva a los trabajadores al buen trabajo, un cuarenta por ciento (40\%) indica que casi nunca se ha sentido motivado por la empresa y diez por ciento (10\%) dice que nunca lo motivan. Resultados que hacen factible la propuesta de esta investigación, pues intenta aumentar la motivación de los trabajadores al ser aplicada por la empresa, pues siempre se debe motivar al personal. Sverdlik (citado por Rodríguez, 2006), como el proceso por el cual se moviliza la conducta y se sostiene en el interés de satisfacer las necesidades individuales y lograr los objetivos organizacionales, lo cual conlleva a aumentar la rentabilidad.

Análisis: Indicador: Objetivos. Según indicó el cincuenta por ciento (50\%) de los encuestados, la empresa casi nunca tiene definido los objetivos, como aspiraciones a lograr en términos de rendimiento y en este mismo orden el cincuenta por ciento (50\%) restante indica que nunca los define. Por ello, se hace necesario que la propuesta de investigación defina estos objetivos, los cuales Gutiérrez (2004) indica que constituyen aspiraciones permanentes a lograr por la organización en términos de rendimiento, pueden ser sobre aspectos como: inversión, liderazgo o posición en el mercado, crecimiento, imagen, productividad y bienestar para sus miembros, entre otros, para que se pueda obtener lo deseado y una mayor calidad en la organización.

Análisis: Indicador: Metas. El cincuenta por ciento (50\%) de los trabajadores indico que casi nuca existe en la empresa mitos y relatos, que son como creencias que muchas veces se tienen y se respetan. El cuarenta por ciento (40\%) dijo que a veces los observa y el resto, diez por ciento (10\%) indicó que nunca existen. Kreitner y Kinicki (1997) señalan que, de los mitos no se sabe a ciencia cierta si son del todo verdaderos, pero a pesar de esto, ejercen autoridad en la empresa e influyen en las percepciones comunes de los empleados. Los mitos son de gran importancia, ya que, si los gerentes saben manejarlos y aprovecharlos, pueden servir de difusión y mantenimiento de los principios organizacionales y los valores.

Análisis: Indicador: Los Rituales y Ceremonias. En este particular, el cien por ciento (100\%) de los trabajadores en estudio indicó que la empresa nunca hace celebraciones por los cumpleañeros del mes o día del trabajador. Estos resultados hacen factible la propuesta de investigación, pues debe promocionarse la celebración de rituales y ceremonias. Puesto que las celebraciones deben realizarse cuando los trabajadores alcanzan puntos importantes en determinados aspectos, pueden ser en sus carreras, producción, antigüedad, entre otros; las ceremonias son parte importante de la cultura, ya que la refuerzan por que proporcionan experiencias que son recordadas con orgullo por el empleado.

Los rituales forman parte importante de una organización, son una serie de rutinas, las cuales son programadas, estandarizadas y sistemáticas, estas rutinas enseñan a los trabajadores los comportamientos que la organización desea que estos tengan. 
Las Ceremonias, Marín L. (1997) las define como el "sistema de ritos conectados con una ocasión o evento singular" (p. 146), estas manifestaciones dan ejemplo de lo que la empresa representa. Estas pueden darse en forma de celebraciones que son realizadas cuando los trabajadores alcanzan puntos importantes en determinados aspectos, pueden ser en sus carreras, producción, antigüedad, entre otros; las ceremonias es una forma utilizada por la organización para festejar los héroes, mitos, entre otros.

Análisis: Indicador: Las Normas: El cien por ciento (100\%) de los trabajadores indicó que nunca existen normas establecidas de comportamiento organizacionales en la empresa C.M., C.A. Lo cual hace evidente su instauración en la empresa para el fortalecimiento de la cultura organizacional. Según Deal y Kennedy (1985) son reglas, pautas establecidas dentro de la organización, los gerentes a través de la cultura y dependiendo del manejo que a esta se le dé, puede influir en la manera de actuar de los trabajadores, esto lo puede hacer mediante las normas establecidas.

Análisis Indicador: Las Políticas. En este particular, el cien por ciento (100\%) de los trabajadores señalo que en la empresa nunca existen planes que establezcan las políticas de la empresa. Lo cual constituye, una debilidad para la empresa, puesto que es necesaria la implementación de políticas empresariales. Ya que, Gutiérrez (2004) instituye que las políticas generales de una empresa, establecen su normativa de acción, constituyen pautas, métodos, procedimientos, reglas, formas $y$ prácticas administrativas determinadas, que se expresan para estimular y apoyar el trabajo hacia las metas establecidas.

\section{CONCLUSIONES}

El objetivo del estudio es evaluar la cultura organizacional de la empresa C.M., C.A. ubicada en Valencia, estado Carabobo, Venezuela, con la finalidad de recomendar estrategias dirigidas a mejorar el clima y desempeño de la organización. Para lograr esto se definieron como objetivos específicos Diagnosticar la cultura organizacional de la empresa y examinar la cultura existente de la misma.

Al diagnosticar la cultura organizacional de la empresa C.M., C.A., se evidenció que en la empresa el comportamiento laboral está influenciado por el entorno y los factores externos siempre tienen incidencia en su rendimiento; la empresa no posee valores, misión, visión, objetivos, mitos y relatos, metas, normas ni políticas definidas. Tampoco se reconoce ningún empleado líder y promotor de buenas acciones; los trabajadores no tienen sentido de pertenencia para la empresa; la comunicación es poca fluida en la relación entre estos; y los trabajadores están poco motivados al buen trabajo y la empresa nunca hace celebraciones por los cumpleañeros del mes o día del trabajador.

De acuerdo al análisis se puede decir que en la empresa de estudio, la cultura organizacional es inexistente, aunque al haber observado el desarrollo de las operaciones día a día, se parece mucho al tipo de cultura definida por Deal y Kennedy (1985) como cultura del proceso: Se caracteriza por un clima de riesgo bajo y retroalimentación lenta que permite realizar cierta predicción de los hechos y prepararse para ello, por lo que la tensión se concentra en el cómo hacer el trabajo y no en el qué. Las personas que trabajan en esta cultura tienden a desarrollar una mentalidad de protegerse la espalda, en donde el más mínimo acontecimiento amerita una amonestación. 
De acuerdo a los resultados del estudio se puede decir que uno de los retos más importantes a los que se enfrenta la empresa, es el de desarrollar y/o fomentar una cultura organizacional sólida enmarcada en la instauración de valores, creencias, y sentimientos que logren crear en los trabajadores sentido de pertenencia, de trabajo en equipo, de compromiso con la empresa, en fin, de una serie de aspectos que van en pro de optimizar las capacidades de liderazgo tanto de la gerencia como de los trabajadores en general

La cultura organizacional es valiosa tanto para la organización como para los trabajadores, ya que esta intensifica el compromiso organizacional e incrementa la consistencia del comportamiento del empleado y es evidente que estos son beneficios para la organización, también desde el punto de vista de los empleados, la cultura es valiosa, porque reduce la ambigüedad, pero no se debe olvidar, según Robbins, (2004) que existen "algunos aspectos de la cultura que pueden ser disfuncionales y que pueden afectar la eficacia de la organización" ( $p$. 123). Por lo tanto, se recomienda el diseño de estrategias para afianzar la cultura organizacional, en la empresa C.M., C.A. ubicada en Valencia estado Carabobo, descritas como:

- Definir la misión y visión de la empresa. Establecer metas, objetivos, normas y políticas organizacionales.

- Fortalecer valores a través de actividades de crecimiento personal. Fortalecer la identidad institucional y sentido de pertenencia. Definir un sistema comunicación con los trabajadores.

- Reconocer la labor del empleado líder. Hacer acompañamiento a los trabajadores en el desarrollo de las operaciones y celebraciones. Motivar la labor del trabajador.

- Capacitar al trabajador.

- Promover actividades socio-culturales junto a la comunidad.

Estas estrategias permitirán aumentar el compromiso e incrementar la consistencia del comportamiento del empleado que consecuentemente defina una cultura organizacional en la empresa C.M., C.A. logrando: Aumentar el sentido de pertenencia, Lograr intereses comunes, Aumentar la socialización con el trabajador y en general coordinar a la organización, puesto que la cultura se encarga de guiar y moldear las actividades, por medio de las normas, valores y metas definidas.

\section{REFERENCIAS}

Andara, N. (2012) Fortalecimiento de la Cultura Organizacional para el Desarrollo de las Relaciones Interpersonales. Expuesto en la Universidad de Carabobo Programa de Gerencia Avanzada en Educación, como requisito para optar al título de Magíster en Gerencia Avanzada en Educación

Camacho, T. (2008). Propuesta de un Plan Para la Promoción de los Valores Institucionales en el Recurso Humano del Departamento de Egresados, en la Dirección de Información y Control Estudiantil de la Universidad de Carabobo. Presentado la Universidad de Carabobo Facultad de Ciencias Económicas y Sociales Escuela de Relaciones Industriales para optar al Título de Licenciado en Relaciones Industriales

Certo, S. (2009). Administración Moderna. Octava Edición. Bogotá, Colombia: Editorial Pearson Educación

Chiavenato, I. (2000). Introducción a la teoría general de la administración. Quinta edición. Editorial McGraw Hill. México 
Chiavenato, I. (2004). Administración de Recursos Humanos. $\quad 5^{\text {a }}$ Edición. McGraw-Hill Interamericana. México

Daft, R. y Marcic, D. (2006). Introducción a la Administración.(4a ed.). México

Thomson. Deal y Kennedy. (1985), Culturas Corporativas. Ritos y Rituales de la Vida Organizacional

Denison, D. (1991). Cultura Corporativa y Productividad Organizacional. Bogotá. Legis Fondo Editorial

Fred R., D. (2003). La Gerencia Estratégica. Legis Editores S.A. Colombia

García, J. (2011) Estrategias Gerenciales para el Fomento de la Cultura Organizacional. Presentado ante la Dirección de Estudios de Postgrado de la Universidad de Carabobo, para optar al Título de Magíster en Gerencia Educativa.

Gibson, J. (2003). El comportamiento de las organizaciones. Editorial Mc Graw Hill, décima edición. México

Gordon, J. (1997). Comportamiento Organizacional. Prentice Hall Hispanoamericano S.A., México

Gutiérrez, M. (2004). Administrar para La Calidad. ( $2^{a}$ ed.). México: Limusa

Harmon, F. y Garry, J. (1995). Diferencia vital. Bogotá: Norma

Kreitner, R. y Kinicki, A. (1997). Comportamiento de las Organizaciones. Editorial Mc Graw Hill. Tercera. Edición
López M, A. (1999). El Proceso de la Entrevista. Editorial Limusa, S.A de c.v. México. 200p

Marin, L. (1997). Sociología para las empresas. Barcelona, España. Editorial Mc Graw Hill

Mintzberg, H. (2001). El Proceso Estratégico. Ciudad de México, México: Prentice May DF

Ochoa, Y. (2013). La Cultura Organizacional y el Liderazgo como Alternativas para Lograr la Eficiencia en los Servicios Prestados en la Unidad de Atención Médica Integral de la Universidad de Carabobo. Presentado en la Universidad de Carabobo Facultad de Ciencias Económicas y Sociales Área de Postgrado, para optar al grado de Magíster en Administración de Empresas, Mención Gerencia.

Pérez S., G. (2002). Investigación Cualitativa: Retos e interrogantes.Técnicas y Análisis de los Datos. Colección Aula Abierta. Editorial La Muralla, S.A. Segunda Edición.

Robbins, S. (2004). Comportamiento Organizacional. Editorial Pearson Prentice Hall. 10ma Edición. México

Rodríguez, M. (2006). La Importancia de la Formación en el ámbito Empresarial Actual. Editorial Ideas Propias. España

Rosenzweig, D. y Kast, A. (2000). Administración en las organizaciones. Un enfoque de sistemas. México. Editorial Mc Graw Hill 